\title{
OPTIMIZATION OF POSITIONING OF GROUND STATIONS FOR SPACE OPTICAL MISSIONS
}

\author{
B. Grishechkin, A. Braun, M. Wickler \\ German Space Operations Center of German Aerospace Center (DLR)
}

\begin{abstract}
Every space mission which uses optical band, e.g. groundsatellite/satellite-ground laser telecommunication, optical earth observation, on-ground optical space debris tracking system, is drastically affected by the clouds in the troposphere of the Earth. Mission planning group of the German Space Operations Center (GSOC) is investigating the possibility of achieving the maximum performance of future optical space missions by involving cloud cover information (CI).
\end{abstract}

\section{INTRODUCTION}

An important part of each mission operated by GSOC, especially of an earth observation mission, is a Mission Planning System. Its task is collecting and analyzing of information, availabilities and requirements that are relevant to the on-board operations [1].

For future missions which use optical band, e.g. optical Earth remote sensing mission or a laser telecommunication mission, a new component of MPS which reads and processes relevant meteorological data have to be used, it is called further cloud information software (CIS). The CIS is developed and described fully in [2], below is a short description. The "GRIB file" input of CIS, see Figure 1, is of a standard format [3]. The total cloud cover based on the CI from a provider like DWD (Germany), ECMWF (UK), NOAA (USA) or Weatheroffice (Canada) recalculated for a particular line ground station - satellite for each particular point in time where the satellite's orbit is given to the optical visibility tool (OVC-tool). The OVCtool can generate the timetable for the given ground stations or decide which ground stations are worth of building/ordering if a limited number of stations is given.

\section{SHORT DESCRIPTION OF CIS and OVCtool}

The Optical Visibility Calculator, see Figure 2, takes each given point of the satellite's orbit, calculates the line satellite - ground station and gets from CIS cloud cover for several point on the line for several altitudes within the specified thickness of the cloud layer, see Figure 2. Then it checks if the calculated cloud cover not higher then the specified limit allowed for the optical link and writes the state of the link (possible/impossible) into the timetable. The Decision Maker basing on by user chosen criteria and restrictions on the link, e.g. multiple/single link, the shortest possible link duration, if requested finds the best combination of the ground stations. The entire process is fulfilled for the whole period for which the $\mathrm{CI}$ is given.

\section{PECULARITY OF USING CLOUD INFORMATION}

Basing on our experience [2] of working with CI from NOAA and ECMWF we would like to notice that it is recommended if applicable to discuss some standard parameters of the format before ordering the data, e.g. compression type, coordinate grid type. Though the standard is pretty large and informative it is also necessary to ask the provider for additional description which meteorological data is in there, which physical meaning it has and how it should be used for your needs. The most information in the standard [3] is positioning of the data within a file but not describing of the physical meaning of a parameter.

Our results in [2] also show that the given wind does not define the clouds' movement, this was also noticed independently by another team [4]. We experienced that the wind and the clouds' movement can even have opposite directions. However this could be because of a great difference of the altitudes of given clouds and wind in the files we used. In our case the level at which the total cloud cover was given was "entire atmosphere (considered as a single layer)" and level at which the wind was given was "specified (10 meter)".

\section{EXAMPLE OF OPTIMIZATION OF GROUND STATIONS POSITIONING}

To calculate the visibility of on-ground stations from a satellite the orbit of the TerraSAR-X satellite was used as an example in this work. There were 4 on-ground stations taken into account, the coordinates of the used stations were taken approximately in according with the map shown in the article [5], i.e. the northeast, northwest, southeast and southwest corners of Germany $\left(\left(7.2^{\circ} ; 53.5^{\circ}\right),\left(13.5^{\circ} ; 53.5^{\circ}\right)\right.$, $\left.\left(7.2^{\circ} ; 47.7^{\circ}\right),\left(13.5^{\circ} ; 47.7^{\circ}\right)\right)$, see Figure 3 . The period of CI taken into account here was between 13/08/1995 and 


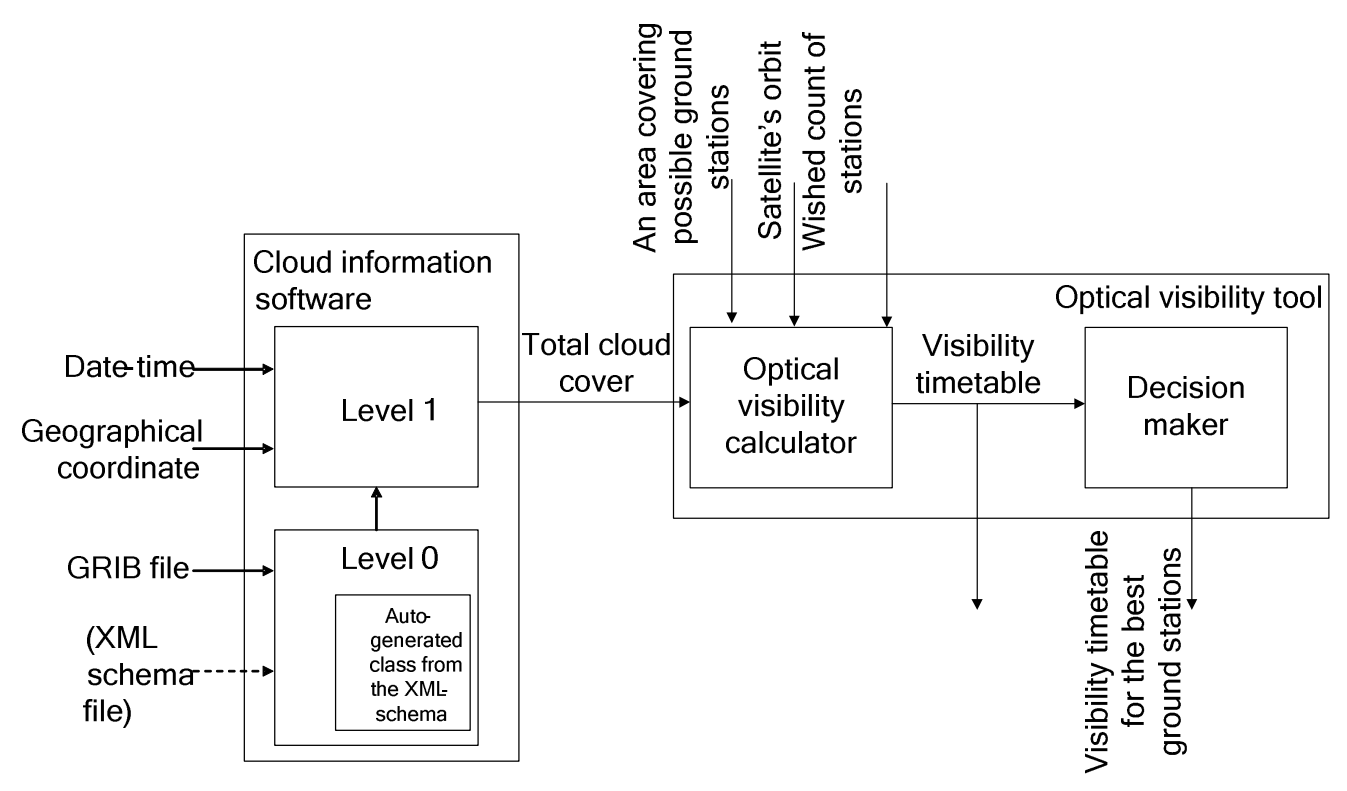

Figure 1. The structure of CIS and OVCtool

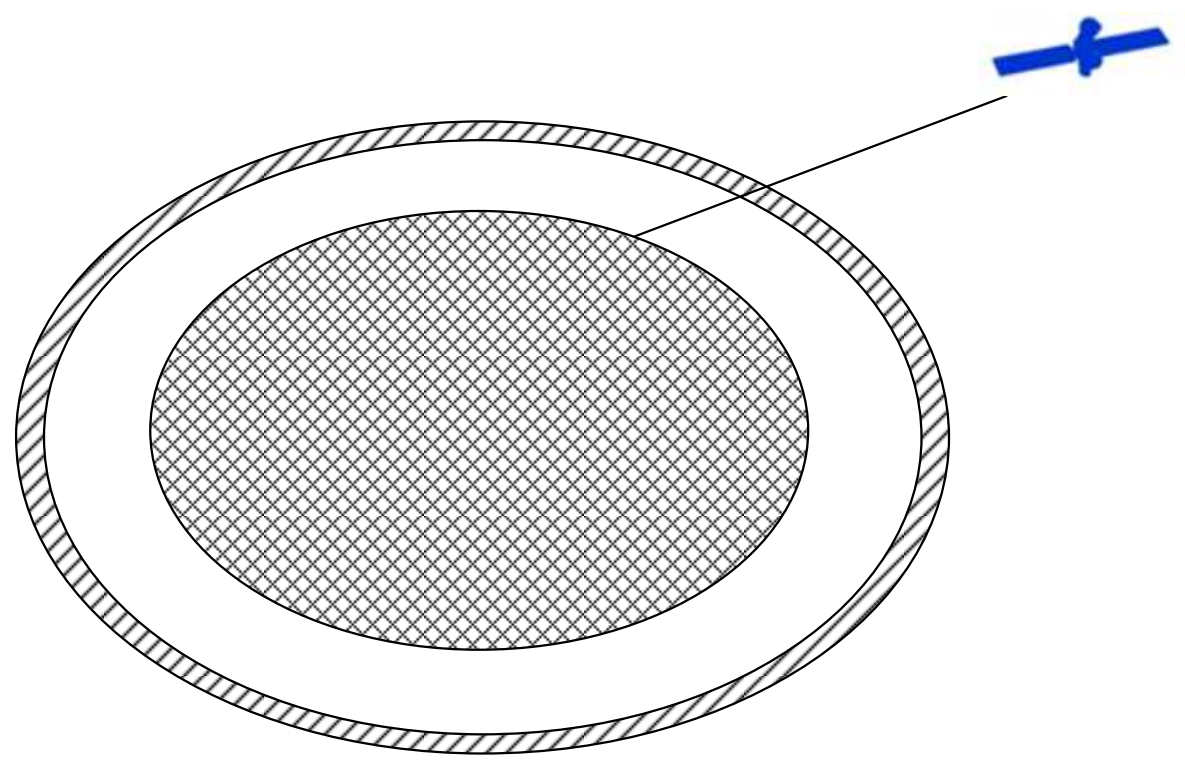

Figure 2. The line ground station - satellite crossing the cloud layer

$31 / 12 / 1996$, i.e. slightly longer than one year. The cloud coverage from GRIB files was presumed to be given between the sea-level and the upper level of the troposphere, i.e. $17 \mathrm{~km}$. The simulation showed that within the taken period there are 81430 seconds during which the satellite is above at least one of the on-ground stations (elevation is more than $40^{\circ}$ ) which has a cloudless link with satellite, see the first line in Table 1. Cloudless here means that the total cloud coverage is less than $30 \%$. One can refine these results if some parameters of a particular laser telecommunication system e.g. highest allowed cloud coverage, link set time and the possible shortest link duration are taken into account. We assumed here also that the satellite can have a link with only one station at the same time.

According to the calculation method above the presented in Figure 3 positioning of the on-ground stations was 


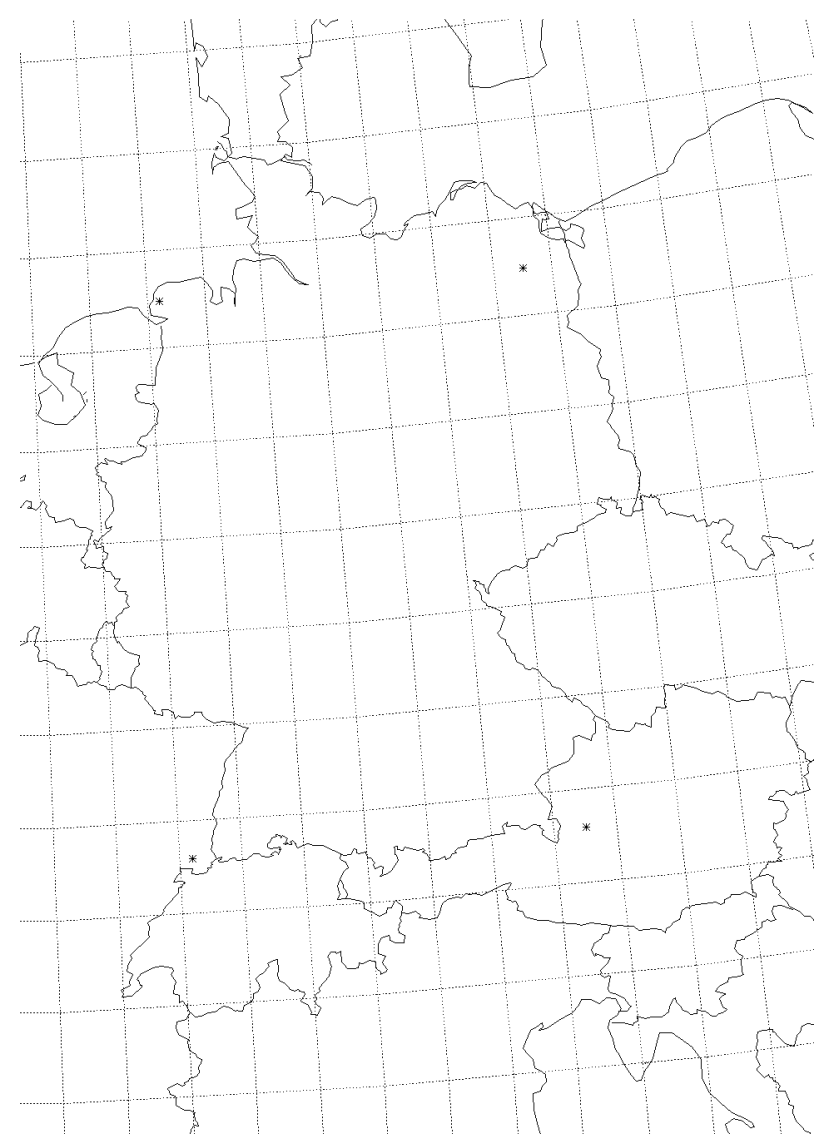

Figure 3. Default positioning of on-ground stations

optimized by the criteria of getting the longest overall link duration while having on-ground stations inside the box defined by the default stations. The optimized positioning of the on-ground stations is shown in Figure $4\left(\left(9^{\circ} ; 53.5^{\circ}\right)\right.$, $\left.\left(13.5^{\circ} ; 53.5^{\circ}\right),\left(9^{\circ} ; 48.5^{\circ}\right),\left(8^{\circ} ; 49^{\circ}\right)\right)$. This optimization raised the overall link duration by about $10 \%$. According to the features of a particular mission one can also choose another criteria and execute the corresponding optimization.

\section{CONCLUSION}

Optimization of positioning of on-ground stations for an optical mission as well as assessing its performance is possible by using CI from GRIB files. In the shown example the overall time link duration of a satellite-earth/earthsatellite laser telecommunication system was raised at least by $10 \%$. There are some other algorithms which can be used or be a base to implement optimization of the ground stations positioning [6-9] and there can be also a "bruteforce" algorithm implemented where all possible combinations of given number of stations are to be compared. The continuation of the work is also implementing of the capability to define the area of possible ground stations as a polygon and the possibility to input

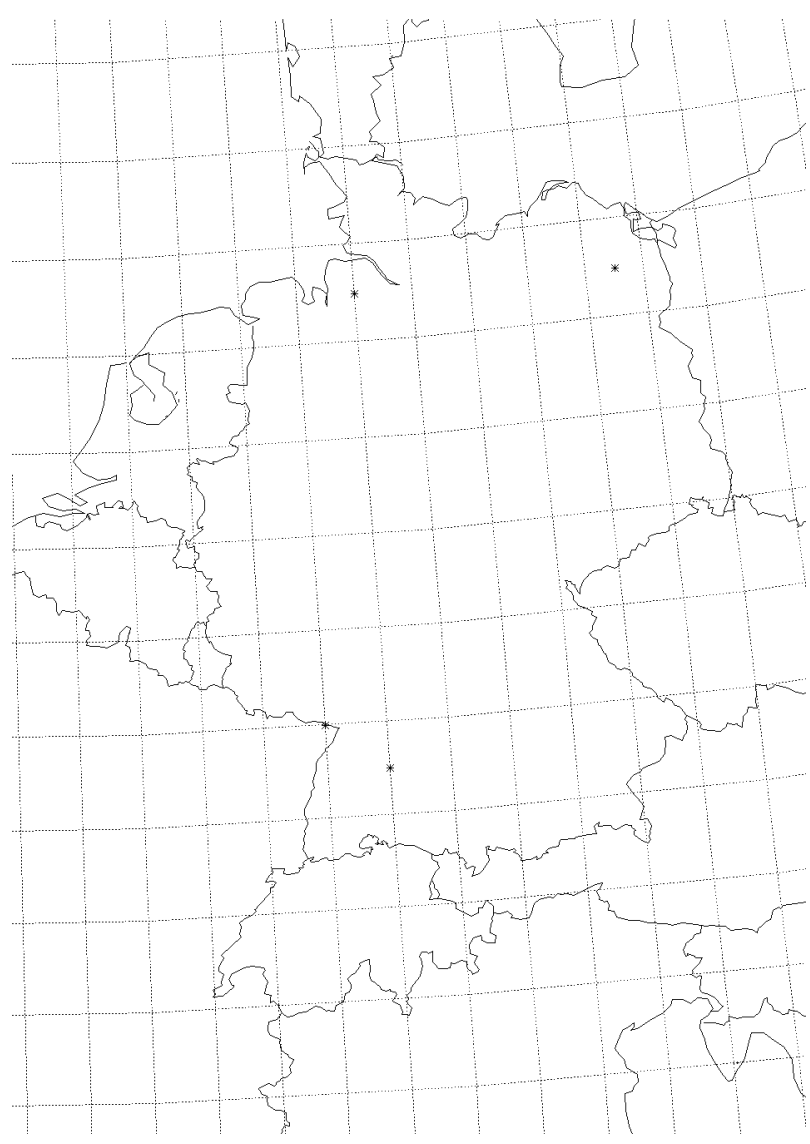

Figure 4. Optimized positioning of on-ground stations

coordinates within the mentioned area where it is not possible to build/order a ground station.

\section{REFERENCES}

[1] M. Wickler, A Mission Planning Concept and Mission Planning System for Future Manned Space Missions, Proceedings of SpaceOps 1994 [online publication], URL: http://www.op.dlr.de/SpaceOps/spops94/wickler/wickler.htm [cited 26 April 2012].

[2] B. Grishechkin, A. Braun, M. Wickler, "Clouds Handling for Planning of Optical Space Missions", Proceedings of SpaceOps 2012.

[3] WMO PUBLICATION No. 306 - MANUAL ON CODES, Volume I.2, World Meteorological Organization, 2010.

[4] CineSat, Frequently asked questions about CineSat, URL: http://www.cinesat.com/csfaq.htm\#NWPWinds [cited 26 April 2012].

[5] C. Fuchs et al., Verification of Ground Station Diversity for Direct Optical TTC-downlinks from LEO Satellites by Means of an Experimental Laser-source, 5th ESA International Workshop on Tracking, Telemetry and Command Systems for Space Applications, 21 - 23 September 2010.

[6] Norman M. Sadeh, Mark S. Fox, Variable and Value Ordering Heuristics for the Job Shop Scheduling Constraint Satisfaction 
Problem, technical report CMU-PJ-TR-91-23, The Robotics Institute Carnegie Mellon University, Pittsburg, November 1995.

[7] Lara S. Crawford, Victor H.L. Cheng, Near-Optimal Antenna Placement Using Genetic Search, American Institute of Aeronautics and Astronautics (AIAA-2000-4914), pp. 1 - 11, 2000 [8] F. Lacoste, A. Guérin, A. Laurens, G. Azema, C. Periard, D. Grimal, FSO Ground Network Optimization and Analysis Considering the Influence of Clouds, Proceeding of EuCAP, Convened Papers, pp. 2746 - 2750, 2011.

[9] Robert A. Morris, John L. Bresina, Stuart M. Rodgers, Automatic Generation of Heuristics for Scheduling, Planning and scheduling, pp. 1260 - 1266, 1997. 